\title{
OPPORTUNISTIC MICROORGANISMS IN PATIENTS UNDERGOING ANTIBIOTIC THERAPY FOR PULMONARY TUBERCULOSIS
}

\author{
Silvia Maria Rodrigues Querido ${ }^{1}$,Graziella Nuernberg Back-Brito ${ }^{1}$, Silvana Soléo Ferreira dos Santos ${ }^{2}$, Mariella Vieira \\ Pereira Leão $^{2^{*}}$, Cristiane Yumi Koga-Ito ${ }^{1}$, Antonio Olavo Cardoso Jorge ${ }^{1}$ \\ ${ }^{1}$ Laboratório de Microbiologia, Departamento de Biociências e Diagnóstico Bucal, Faculdade de Odontologia, São José dos \\ Campos, Universidade Estadual Paulista, São José dos Campos, SP, Brasil; ${ }^{2}$ Laboratório de Microbiologia, Instituto de \\ Biociências Básica, Universidade de Taubaté, Taubaté, SP, Brasil.
}

Submitted: November 08, 2010; Returned to authors for corrections: February 25, 2011; Approved: May 30, 2011.

\begin{abstract}
Antimicrobial therapy may cause changes in the resident oral microbiota, with the increase of opportunistic pathogens. The aim of this study was to compare the prevalence of Candida, Staphylococcus, Pseudomonas and Enterobacteriaceae in the oral cavity of fifty patients undergoing antibiotic therapy for pulmonary tuberculosis and systemically healthy controls. Oral rinsing and subgingival samples were obtained, plated in Sabouraud dextrose agar with chloramphenicol, mannitol agar and MacConkey agar, and incubated for 48 $\mathrm{h}$ at $37^{\circ} \mathrm{C}$. Candida spp. and coagulase-positive staphylococci were identified by phenotypic tests, $C$. dubliniensis, by multiplex PCR, and coagulase-negative staphylococci, Enterobacteriaceae and Pseudomonas spp., by the API systems. The number of Candida spp. was significantly higher in tuberculosis patients, and C. albicans was the most prevalent specie. No significant differences in the prevalence of other microorganisms were observed. In conclusion, the antimicrobial therapy for pulmonary tuberculosis induced significant increase only in the amounts of Candida spp.
\end{abstract}

Key words: tuberculosis; Candida; Enterobacteriaceae; Pseudomonas spp; Staphylococcus.

\section{INTRODUCTION}

Tuberculosis is a chronic infectious disease caused by Mycobacterium tuberculosis or other pathogenic mycobacteria of the Mycobacterium tuberculosis complex. The disease continues to be an important public health problem in various countries. Infection with human immunodeficiency virus
(HIV), injection drug use, increased poverty, migration, malnutrition and urbanization have contributed to the increase in the number of tuberculosis cases worldwide (9). As mycobacteria are able to develop resistance to single-drug regimens, the treatment of choice is based on the use of multiple antimicrobial agents administered over a period of 6 to 12 months (25). 
There is evidence indicating that the long-term use of antibiotics might be related to alterations in the resident oral microbiota and to a possible increase in the occurrence of opportunistic microorganisms (22), as enteric bacilli, Pseudomonas, Staphylococcus and yeasts $(7,19)$.

Candida spp. is present in the oral cavity and is considered a commensal fungi. C. albicans is the predominant species, accounting for 60 to $70 \%$ of all isolations, followed by C. tropicalis and C. glabrata $(2,12,26)$. The incidence of Candida spp. in the oral cavity and the reasons for the establishment of infections caused by these microorganisms have been associated to immunosuppression, endocrine disorders, mucosal lesions, poor oral hygiene, and long-term treatment with antibiotics or corticosteroids $(3,13)$.

Bacteria of the families Enterobacteriaceae and Pseudomonas spp. have been extensively studied and, although these bacteria are not considered pathogenic in healthy human, the oral cavity may serve as a reservoir for these microorganisms and may seriously compromise the life of debilitated individuals $(19,10,23)$.

Microorganisms of the genus staphylococci are not considered part of the resident human oral microflora, but may act as opportunistic pathogens in patients undergoing prolonged systemic treatment with antimicrobial agents or immunosuppressors $(1,17)$.

Several lines of evidence indicate that the prolonged use of antibiotics and host-related factors are associated with alterations in the resident oral microbiota and with a possible increase in the occurrence of opportunistic microorganisms. Therefore, the objective of the present study was to compare the prevalence of Candida, Enterobacteriaceae, Staphylococcus and Pseudomonas in the oral cavity and gingival sites in the oral cavity of patients undergoing antibiotic therapy for pulmonary tuberculosis and healthy controls.

\section{MATERIALS AND METHODS}

The study was approved by the Ethics Committee of the
São José dos Campos Dental School, Univ. Estadual Paulista/UNESP (protocol number 089/2003-PH/CEP). All subjects signed informed consent to participate in the study.

Fifty patients (17 females and 33 males) aged 20 to 73 years with diagnosis of pulmonary tuberculosis, who had undergone antibiotic therapy at the Day Hospital of the Taubaté Medical School for at least 45 days, were included in the study. HIV-positive patients, diabetics, patients with any other systemic diseases, pregnant women, smokers, and patients using dentures or orthodontic devices were excluded.

The control group consisted of 50 healthy subjects aged 20 to 73 years, who were matched for age, gender, denture use and oral conditions to the pulmonary tuberculosis patients, were selected among patients under treatment at the São José dos Campos Dental School. The same exclusion criteria were applied to the tuberculosis patients. In addition, individuals treated with antimicrobial/antifungal agents over the 60 days preceding sample collection were excluded. The mean age was 41.94 years $( \pm 15.29)$ in the tuberculosis group and 42.1 years $( \pm 15.28)$ in the control group.

Samples were obtained from each subject by oral rinses with $0.1 \mathrm{M}$ phosphate-buffered saline (PBS), $\mathrm{pH} 7.2$, for $1 \mathrm{~min}$. The oral rinse samples were centrifuged for $10 \mathrm{~min}$ at $8,000 \mathrm{~g}$ and the supernatant was discarded. Next, $2.5 \mathrm{ml}$ PBS was added to the pellet and dilutions of $10^{-1}$ and $10^{-2}$ were prepared in PBS.

Subgingival samples were also collected with sterile paper points introduced into the bottom of the pocket and kept in place for $30 \mathrm{~s}$ (Maillefer Instruments, Ballaigues, Switzerland). Three samples were collected from each subject and placed into an Eppendorf tube containing $1 \mathrm{ml}$ PBS. The samples were homogenized by vortexing for $30 \mathrm{~s}$ and the paper points were removed with sterile tweezers.

Aliquots $(0.1 \mathrm{ml})$ of each suspension (oral rinses and subgingival biofilm) were plated onto Sabouraud dextrose agar (Difco, Detroit, MI) supplemented with chloramphenicol (0.1 $\mathrm{mg} / \mathrm{ml}$ culture medium), mannitol agar (Difco), and 
MacConkey agar (Difco). The plates were incubated for $48 \mathrm{~h}$ at $37^{\circ} \mathrm{C}$. After growth, the colonies were examined regarding morphological features and characteristic colonies were counted to calculate the number of colony-forming units (CFU) per milliliter. For each colony with different morphology, microscopic confirmation was performed.

For the obtaining of pure cultures, colonies suggestive of yeast were inoculated into test tubes containing Sabouraud dextrose agar and incubated for $24 \mathrm{~h}$ at $37^{\circ} \mathrm{C}$. Colonies of Gram-positive cocci and Gram-negative bacilli were inoculated into test tubes containing gelose agar and incubated for $24 \mathrm{~h}$ at $37^{\circ} \mathrm{C}$. These samples were stored at $4^{\circ} \mathrm{C}$ until identification.

Phenotypic identification of Candida spp. consisted of the determination of germ tube formation in bovine serum, growth on corn meal-Tween 80 agar (Difco), fermentation, and carbohydrate assimilation $(18,24)$. C. albicans reference strain ATCC 18804 was used as control. Isolates identified phenotypically as $C$. albicans/C. dubliniensis were submitted to molecular detection of $C$. dubliniensis. These isolates were analyzed by the polymerase chain reaction (PCR) according to the method of Donnelly et al. (6) and Mähnss et al. (16), with modifications. Briefly, the isolates were plated onto Sabouraud dextrose agar and incubated for $24 \mathrm{~h}$ at $37^{\circ} \mathrm{C}$. Next, a single colony was transferred to $75 \mu \mathrm{L}$ zymolyase solution (Sigma, St. Louis, MO; $0.5 \mathrm{mg} / \mathrm{ml}$ in $1 \mathrm{M}$ sorbitol buffer). The tubes were incubated at $95^{\circ} \mathrm{C}$ for $10 \mathrm{~min}$. The samples were then centrifuged at $8,000 \mathrm{~g}$ for $15 \mathrm{~min}$ at $4^{\circ} \mathrm{C}$ and the supernatant was kept on ice until the time for PCR analysis.

Two pairs of primers were used for PCR: two universal primers, Uni-f: 5'-GCATATCAATAAGCGGAGGAAAA-3' and Uni-r: 5'GGTCCGTGTTTCAAGACG-3', and two $C$. dubliniensis-specific primers, DUBF Act-f: 5'GTATTTGTCG TTCCCCTTTC-3' and DUBR Act-r: 5'-GTGTTGTGTGCAC TAACGTC-3'. Amplification was carried out in a final volume of $10 \mu \mathrm{L}$ containing $5 \mathrm{pmol}$ of each primer, 5.0 $\mu \mathrm{L}$ PCR Master Mix (Promega), $3.2 \mu \mathrm{L}$ ultrapure water, and $1 \mu \mathrm{L}$ of the DNA template. Cycling conditions were $3 \mathrm{~min}$ at $95^{\circ} \mathrm{C}$, followed by
30 cycles of $30 \mathrm{~s}$ at $95^{\circ} \mathrm{C}, 30 \mathrm{~s}$ at $58^{\circ} \mathrm{C}, 60 \mathrm{~s}$ at $72^{\circ} \mathrm{C}$, and a final extension at $72^{\circ} \mathrm{C}$ for $10 \mathrm{~min}$. C. albicans (ATCC 18804) and C. dubliniensis (CD33) reference strains were included as controls in all reactions. The amplification products were separated by electrophoresis on $2 \%(\mathrm{w} / \mathrm{v})$ agarose gels containing $25 \mu \mathrm{M}$ ethidium bromide and visualized under a UV transilluminator (Foto/UV 26, Fotodyne, Inc.). A 100-bp DNA ladder (Gibco, BRL) was used as molecular size standard. The gels were photographed for documentation (DC290 Kodak Photo Documentation System).

Staphylococcus spp. isolates were tested for catalase and coagulase production. Positive strains were identified by acid production from D-trehalose and the Voges-Proskauer test. Coagulase-negative staphylococci were identified using the API Staph system (bioMérieux, France). The Enterobacteriaceae and Pseudomonas spp. isolates were identified using the API 20E system (bioMérieux).

The results were compared statistically by ANOVA and the Mann-Whitney test, at the level of significance of $5 \%$.

\section{RESULTS}

At the time of sample collection, 19 (38\%) patients of the tuberculosis group were in the first phase of treatment, receiving a combination of rifampicin, isoniazid and pyrazinamide, and $31(62 \%)$ were in the second phase of treatment, receiving a combination of rifampicin and isoniazid.

Candida spp. was more prevalent in the oral cavity of the tuberculosis patients, than control individuals, for both analyzed samples (Table 1). Candida counts were also significantly higher in the oral cavity (3.61 log CFU/ml) and gingival sites $(2.62 \log \mathrm{CFU} / \mathrm{ml})$ of patients with tuberculosis, compared to controls (3.16 $\log \mathrm{CFU} / \mathrm{ml}$ and 0,69 $\log \mathrm{CFU} / \mathrm{ml}$, respectively) ( $\mathrm{p}=0.0085$ and $\mathrm{p}=0.0103$, respectively).

From Candida positive individuals, 73 different strains of the yeasts were isolated from tuberculosis patients and 33 , from the control group. C. albicans was the most frequently isolated species in both groups, followed by $C$. tropicalis. A greater 
diversity of Candida isolates was observed in the tuberculosis group compared to the control subjects (Table 2). $C$. dubliniensis was not detected in both groups.

The prevalence of Staphylococcus spp. was slightly higher in the oral cavity of tuberculosis group. In the gingival sites, Staphylococcus spp. was more prevalent among control subjects than among patients with tuberculosis (Table 1). No significant differences in the CFU/ml of Staphylococcus spp. in the oral cavity $(p=0.3664)$ or gingival sites $(p=0.5404)$ were observed between studied groups, with mean counts of 3.43 and 2,39 $\log \mathrm{CFU} / \mathrm{ml}$ in the tuberculosis group and 3.44 and $1,21 \log \mathrm{CFU} / \mathrm{ml}$ in the control group. As many individuals presented more than one strain in the oral cavity, i.e. an association of them, a total of 191 strains of Staphylococcus spp. were isolated, $95(49,7 \%)$ from tuberculosis patients and $96(50,3 \%)$ from control group. Out of them, 40,3\% were coagulasis positive and 59,7\% were coagulasis negative. All the species and their frequency are shown in Table 3.

Enterobacteriaceae and Pseudomonas were also slightly more detected in the oral cavity and gingival sites of patients with tuberculosis (Table1). No significant differences in the mean number of $\mathrm{CFU} / \mathrm{ml}$ of Enterobacteriaceae and Pseudomonas in the oral cavity $(\mathrm{p}=0.1648)$ or gingival sites $(p=0.2879)$ were observed between the tuberculosis and control groups. A total of 125 strains of Enterobacteriaceae and Pseudomonas were isolated, 74 (59,2\%) from tuberculosis patients and $51(40,8 \%)$ from control group. The species and their frequency can be observed in Table 4.

Table 1. Number and percentage of individuals positive for Candida spp., Staphylococcus spp., Enterobacteriaceae and Pseudomonas spp. isolated from the oral cavity and gingival sites, from tuberculosis $(\mathrm{n}=50)$ and control $(\mathrm{n}=50)$ groups. Total (all individuals, $\mathrm{n}=100)$.

\begin{tabular}{lcccc}
\hline & \multicolumn{2}{c}{ Oral cavity } & \multicolumn{2}{c}{ Gingival sites } \\
\hline & $\begin{array}{c}\text { Tuberculosis } \\
\mathbf{n}(\mathbf{\%})\end{array}$ & $\begin{array}{c}\text { Control } \\
\mathbf{n}(\mathbf{\%})\end{array}$ & $\begin{array}{c}\text { Tuberculosis } \\
\mathbf{n}(\mathbf{\%})\end{array}$ & $\begin{array}{c}\text { Control } \\
\mathbf{n}(\mathbf{\%})\end{array}$ \\
\hline Candida spp. & $36(72)$ & $26(52)$ & $19(38)$ & $05(10)$ \\
\hline Staphylococcus spp. & $48(96)$ & $46(92)$ & $19(38)$ & $22(44)$ \\
\hline Enterobacteriaceae e Pseudomonas spp. & $28(56)$ & $24(48)$ & $09(18)$ & $05(10)$ \\
\hline
\end{tabular}

Table 2. Percent distribution of Candida species isolated from patients with tuberculosis $(n=50)$ and controls $(n=50)$.

\begin{tabular}{lcc}
\hline Species & $\begin{array}{c}\text { Tuberculosis } \\
\text { n } \mathbf{( \% )}\end{array}$ & $\begin{array}{c}\text { Control } \\
\mathbf{n}(\%)\end{array}$ \\
\hline C. albicans & $31(42,5)$ & $26(78,8)$ \\
C. tropicalis & $21(28,8)$ & $7(21,2)$ \\
C. parapsilosis & $5(6,8)$ & $0(0)$ \\
C. stellatoidea & $4(5,5)$ & $0(0)$ \\
C. lipolytica & $3(4,1)$ & $0(0)$ \\
C. krusei & $3(4,1)$ & $0(0)$ \\
C. glabrata & $2(2,7)$ & $0(0)$ \\
C. kefyr & $2(2,7)$ & $0(0)$ \\
C. guilliermondi & $1(1,4)$ & $0(0)$ \\
C. famata & $1(1,4)$ & $0(0)$ \\
\hline Total & $73(100)$ & $33(100)$ \\
\hline
\end{tabular}


Table 3. Percent distribution of staphylococci species $(n=191)$ detected in the oral cavity of patients with tuberculosis and control individuals.

\begin{tabular}{llcc}
\hline Species & Coagulasis & $\begin{array}{c}\text { Tuberculosis } \\
\mathbf{n}(\mathbf{\%})\end{array}$ & $\begin{array}{c}\text { Control } \\
\mathbf{n}(\mathbf{\%})\end{array}$ \\
\hline S. aureus & positive & $25(26,3)$ & $32(33,3)$ \\
S. schleiferi & positive & $3(3,2)$ & $17(17,7)$ \\
S. epidermidis & negative & $32(33,7)$ & $26(27,1)$ \\
S. warneri & negative & $8(8,4)$ & $2(2,1)$ \\
S. capitis & negative & $6(6,3)$ & $2(2,1)$ \\
S. haemolyticus & negative & $4(4,2)$ & $3(3,1)$ \\
S. xylosus & negative & $3(3,2)$ & $2(2,1)$ \\
S. saprophyticus & negative & $3(3,2)$ & $1(1,0)$ \\
S. lugdunensis & negative & $1(1,1)$ & $4(4,2)$ \\
S. sciuri & negative & $1(1,1)$ & $3(3,1)$ \\
S. hominis & negative & $1(1,1)$ & $2(2,1)$ \\
S. lentus & negative & $2(2,1)$ & $2(2,1)$ \\
S. cohnii ureal & negative & $2(2,1)$ & $0(0)$ \\
S. auricularis & negative & $2(2,1)$ & $0(0)$ \\
S. simulans & negative & $1(1,1)$ & $0(0)$ \\
S. chromogenes & negative & $1(1,1)$ & $0(0)$ \\
\hline Total & & $95(100)$ & $96(100)$ \\
\hline
\end{tabular}

Table 4. Percent distribution of Enterobacteriaceae and Pseudomonas species $(n=125)$ detected in the oral cavity of patients with tuberculosis and control individuals.

\begin{tabular}{ccc}
\hline Species & $\begin{array}{c}\text { Tuberculosis } \\
\mathbf{n}(\mathbf{\%})\end{array}$ & $\begin{array}{c}\text { Control } \\
\mathbf{n}(\mathbf{\%})\end{array}$ \\
\hline Enterobacter cloacae & $16(21,6)$ & $10(19,6)$ \\
Klebsiella oxytoca & $4(5,4)$ & $8(15,7)$ \\
Chryseomonas luteola & $8(10,8)$ & $2(3,9)$ \\
Klebsiella pneumoniae & $5(6,8)$ & $4(7,8)$ \\
Enterobacter sakazakii & $4(5,4)$ & $4(7,8)$ \\
Serratia liquefaciens & $6(8,1)$ & $1(2,0)$ \\
Escherichia coli & $4(5,4)$ & $1(2,0)$ \\
Citrobacter freundii & $2(2,7)$ & $2(3,9)$ \\
Serratia ficaria & $3(4,1)$ & $2(3,9)$ \\
Enterobacter asburiae & $2(2,7)$ & $2(3,9)$ \\
Serratia marcescens & $4(5,4)$ & $0(0)$ \\
Klebsiella ornithinolytica & $1(1,4)$ & $2(3,9)$ \\
Pantoea spp. & $1(1,4)$ & $3(5,9)$ \\
Serratia odorifera & $2(2,7)$ & $1(2,0)$ \\
Kluyvera spp. & $2(2,7)$ & $1(2,0)$ \\
Enterobacter aerogenes & $1(1,4)$ & $1(2,0)$ \\
Pseudomonas aeruginosa & $1(1,4)$ & $1(2,0)$ \\
Pseudomonas fluorescens & $1(1,4)$ & $0(0)$ \\
Burkholderia cepacia* & $1(1,4)$ & $1(2,0)$ \\
Citrobacter koseri & $1(1,4)$ & $1(2,0)$ \\
Salmonella spp. & $1(1,4)$ & $1(2,0)$ \\
Klebsiella terrigena & $1(1,4)$ & $1(2,0)$ \\
Morganella morganii & $1(1,4)$ & $0(0)$ \\
Serratia plymuthica & $1(1,4)$ & $0(0)$ \\
Serratia fonticola & $1(1,4)$ & $0(0)$ \\
Shigella spp. & $0(0)$ & $1(2,0)$ \\
Enterobacter amnigenus & $0(0)$ & $1(2,0)$ \\
\hline Total & $74(100)$ & $51(100)$ \\
\hline & &
\end{tabular}

* previously named Pseudomonas cepacia 


\section{DISCUSSION}

Systemic diseases and conditions such as pulmonary tuberculosis may have direct oral manifestations or exert an indirect effect by modifying the inflammatory or immune response of the host and by altering host-parasite interactions (5). Oral cavity harbors a heterogeneous composition of microorganisms. The subgingival biofilm is a complex bacterial community that adheres to the root surface and is separated from the oral cavity by the soft tissue pocket wall. Under these conditions, many microorganisms with high virulence potential, such as Candida, Staphylococcus and Pseudomonas spp., are able to colonize, grow and cause tissue damage (11). No studies are available in the literature regarding the composition of the oral microbiota or subgingival microflora in patients undergoing antibiotic therapy for pulmonary tuberculosis.

Infections caused by Candida currently represent a significant problem, especially among immunosuppressed patients. In the present study, the prevalence and the amounts of Candida spp. were higher in patients diagnosed with pulmonary tuberculosis undergoing antibiotic therapy, when compared with control group. Besides the compromised immune efficiency of these patients, the antibiotic therapy could have interfered with bacterial microbiota balance, favoring the growth of the yeasts. In the literature, variations in the prevalence of Candida in the oral cavity can be found in different populations, but most of the studies have shown that systemic conditions predispose to higher percentages. BackBrito et al. (2) detected Candida spp. in the oral cavity of $73.3 \%$ of HIV-positive patients; Belazi et al. (3) reported the prevalence of $64 \%$ in patients with type 2 diabetes mellitus, while Kaklamanos et al. (10) found $46.6 \%$ in elderly individuals. In addition to systemic predisposing factors, differences in oral conditions and in the method, sampling sites and sample collection, as well as in demographic and socioeconomic characteristics may explain the differences in the reported frequencies.

Regarding the species identified, $C$. albicans was the most frequently isolated species in both groups, followed by $C$. tropicalis. C. albicans was isolated from $60.8 \%$ of patients with tuberculosis, in agreement with other studies showing that C. albicans is the predominant species in the oral cavity, accounting for 60 to $70 \%$ of all isolations (10), followed by $C$. tropicalis $(21,26)$. However, in contrast to other authors who reported $C$. glabrata to be the third most prevalent species after C. albicans and C. tropicalis, in the present study $C$. parapsilosis was the third most frequent species, isolated from $4.4 \%$ of patients with pulmonary tuberculosis. C. glabrata was detected in only $1.7 \%$ of patients, a rate lower than the 7 to $24 \%$ reported by various studies $(4,26)$.

Another finding of the present study was the greater diversity of Candida species in patients with pulmonary tuberculosis. These results agree with previous studies reporting higher prevalence of non-albicans species in patients with different predisposing factors $(13,2)$. Probably, the imbalance in the microbiota favored the adherence of other species of Candida, which normally would not be able to adhere.

In the present study, the prevalence of Staphylococcus spp. was high in both groups (96\% in the tuberculosis group and $92 \%$ in the control group). These rates are higher than the $61.36 \%$ reported by Loberto et al. (15) for the oral cavity of patients diagnosed with chronic periodontitis. Staphylococcus spp. is a common resident of the nasal microbiota (8) and may reach the mouth of any individual. Ohara-Nemoto et al. (17) provided evidence of this nasal-oral transmission of Staphylococcus species by genotyping (PFGE).

$S$. epidermidis was the most prevalent species in the tuberculosis group, corresponding to $33.7 \%$ of all isolates, followed by $S$. aureus (26.3\%). Loberto et al. (15) also found higher prevalence of $S$. epidermidis in the oral cavity of patients with periodontal disease. However, the most prevalent species in the control group was $S$. aureus (33.3\%), followed 
by $S$. epidermidis $(27.1 \%)$. These data are in accordance to Ohara-Nemoto et al. (17) and Belazi et al. (3) who reported that $S$. aureus was the most frequently isolated bacterial species in saliva (46.4\%) and oral mucosa of patients with dentures $(52.4 \%)$, respectively. There are insufficient available data to determine whether $S$. aureus isolates were colonizing or infecting the oral cavity (Smith et al., 2003). On the other hand, the high prevalence rates of Staphylococcus spp. emphasize the importance of the oral cavity as a significant reservoir of these microorganisms, which might be disseminated to other regions of the body, especially in immunosuppressed patients, or transmitted to other individuals, foods and objects. In a 3-year retrospective study of clinical laboratory data, Smith et al. (20) suggested that $S$. aureus may be more frequently isolated from the oral cavity than suspected.

High prevalence of Enterobacteriaceae and Pseudomonas spp. was also observed in the oral cavity of studied patients with pulmonary tuberculosis (56\%) and healthy controls (48\%). Lower frequency of isolation of Enterobacteriaceae $(20 \%)$ was reported by Kaklamanos et al. (10) studying oral samples from elderly individuals. E. cloacae was the most prevalent species in the tuberculosis group and in the control group. However, Leung et al. (14) found more $K$. oxytoca $(5.3 \%)$ than E. cloacae $(1,6 \%)$ in oral samples obtained from adult subjects (20 to 46 years). Klebsiella oxytoca was the second most frequently isolated species from the oral cavity of control subjects $(16.6 \%)$.

Although higher prevalence of Staphylococcus spp. and enterobacteria were observed in tuberculosis patients, the differences were not significant, as observed with Candida spp. Some patients were receiving a combination of rifampicin, isoniazid and pyrazinamide, and others just rifampicin and isoniazid. Except rifampicin, the other antibiotics are very specific to mycobacteria, having no effect against other groups of bacteria. Probably, that is why the amounts of Staphylococcus spp. and enterobacteria were not affected and were almost the same as control group. However the diversity and frequency of species seemed to be different between the studied groups, suggesting that long-term therapy, especially because of rifampicin, could have induced the selection of resistant strains and consequently a change in microbiota profile. A change in antimicrobial sensitivity of these strains could also have occurred and future investigation in this field could help to find out the ideal treatment of the patients who develop an opportunistic infection by these microorganisms.

Candida spp., Staphylococcus spp., and bacteria from the families Enterobacteriaceae and Pseudomonas spp. are frequently described as potential opportunistic pathogens, presenting high prevalence in the human oral cavity. Within this context, studies on the virulence and pathogenicity of these species and their association with other microorganisms are always important.

In conclusion, the present study suggests that the antimicrobial therapy for pulmonary tuberculosis induced significant increase in the amounts of Candida spp. and changes in diversity of species of all opportunistic microorganisms studied.

\section{ACKNOWLEDGEMENTS}

The authors would like to thank Fundação de Amparo à Pesquisa do Estado de São Paulo (FAPESP, grants 04-10654-9 and 05-55135-1) for financial support.

\section{REFERENCES}

1. Agarwal, R.; Gupta, D.; Ray, P.; Agarwal, .A.; Jindal, S.K. (2006). Epidemiology, risk factors and outcome of nosocomial infections in a respiratory intensive care unit in North India. J. Infect. 53(2), 98-105.

2. Back-Brito, G.N.; Mota, A.J.; Vasconcelos, T.C.; Querido, S.M.R.; Jorge, A.O.; Reis, A.S.; Balducci, I.; Koga-Ito, C.Y. (2009). Frequency of Candida spp. in the oral cavity of Brazilian HIV-positive patients and correlation with CD4 cell counts and viral load. Mycopathologia. 167, $81-87$.

3. Belazi, M.; Velegraki, A.; Fleva, A.; Gidarakou, I.; Papanaum, L.; Baka, D.; Daniilidou, N.; Karamitsos, D. (2005). Candidal overgrowth in 
diabetic patients: potential predisposing factors. Mycoses. 48(3), 192196.

4. Cannon, R.D.; Holmes, A.R.; Mason, A.B.; Monk, B.C. (1995). Oral Candida: clearance, colonization, or candidiasis? J. Dent. Res. 74(5), 1152-1161.

5. Castillo, A.; Mesa, F.; Liébana, J.; García-Martinez, O.; Ruiz, S.; GarcíaValdecasas, J.; O'Valle, F. (2007). Periodontal and oral microbiological status of an adult population undergoing haemodialysis: a cross-sectional study. Oral diseases. 13, 198-205.

6. Donely, S.M.; Sullivan, D.J.; Shanley, D.B.; Coleman, D.C. (1999). Phylogenetic analysis and rapid identification of Candida dubliniensis based on analysis of ACT1 intron and exon sequences. Microbiology. $145,1871-1882$

7. Flynn, M.J.; Slots, J. (1993). Beta-hemolytic streptococci in advanced periodontitis. Oral Microbiol. Immunol. 8(5), 295-297.

8. Güçlü, E.; Yavuz, T.; Tokmak, A.; Behçet, M.; Karali, E.; Öztürk, Ö.; Egeli, E. (2007). Nasal carriage of pathogenic bacteria in medical students: effects of clinic exposure on prevalence and antibiotic susceptibility. Eur. Arch. Otorhinolaryngol. 264, 85-88.

9. Hopewell, P.C. (2002). Tuberculosis control: how the world has changed since 1990. Bull World Health Org. 80(6), 427.

10. Kaklamanos, E.G.; Charalampidou, M.; Menexes, G.; Topitsoglou, V.; Kalfas, S. (2005). Transient oral microflora in Greeks attending Day centres for the elderly and residents in homes for the elderly. Gerodontology. 22, 158-167.

11. Kesavalu, L.; Chandrasekar, B.; Ebersole, J.L. (2002). In vivo induction of proinflammatory cytokines in mouse tissue by Porphyromonas gingivalis and Actinobacillus actinomycetemcomitans. Oral Microbiol. Immunol. 17, 177-180.

12. Koga-Ito, C.Y.; Lyon, J.P.; Vidotto, V.; Resende, M.A. (2006). Virulence factors and antifungal susceptibility of Candida albicans isolates from oral candidosis patients and control individuals. Mycopathologia. 161, 219-223.

13. Komiyama, E.Y.; Ribeiro, P.M.; Junqueira, J.C.; Koga-Ito, C.Y.; Jorge, A.O.C. (2004). Prevalence of yeasts in the oral cavity of children treated with inhaled corticosteroids. Braz. Oral Res. 18(3), 197-201.

14. Leung, W.K.; Yau, J.Y.Y.; Cheung, B.P.K.; Jin, L.J.; Zee, K.Y.; Lo,
E.C.M.; Samaranayake, L.P.; Corbet, E.F. (2003). Oral colonization by aerobic and facultatively anaerobic Gram-negative rods and yeast in Tibetans living Lhasa. Arch. Oral Biol. 48, 117-123.

15. Loberto, J.C.S.; Martins, C.A.P.; Santos, S.S.F.; Jorge, A.O.C. (2004). Staphylococcus spp. in the oral cavity and periodontal pockets of chronic periodontitis patients. Braz. J. Microbiol. 35, 64-68.

16. Mähnss,..B.; Stehr, F.; Schäfer, W.; Neuber, K. (2005). Comparison of standard phenotypic assays with a PCR method to discriminated Candida albicans and Candida dubliniensis. Mycoses. 46, 55-61.

17. Ohara-Nemoto, J.; Haraga, H.; Kimura, S.; Nemoto, T.K. (2008). Occurrence of staphylococci in the oral cavities of healthy adults and nasal-oral trafficking of the bacteria. J. Med. Microbiol. 57, 95-99.

18. Sandvén, P. (1990). Laboratory identification and sensitivity testing of yeast isolates. Acta Odontol. Scand. 48(1), 27-36.

19. Slots, J.; Rams, T.E.; Feik, D.; Taveras, H.D.; Gillespie, G.M. (1991). Subgingival microflora of advanced periodontitis in the Dominican Republic. J. Periodontol. 62(9), 543-547.

20. Smith, A.J.; Robertson, D.; Tang, M.K.; Jackson, M.S.; MacKenzie, D.; Bagg, J. (2003). Staphylococcus aureus in the oral cavity: a three-year retrospective analysis of clinical laboratory data. Br. Dent. J. 195, 701703 .

21. Stenderup, A. (1990). Oral mycology. Acta Odontol. Scand. 48(1), 3-10.

22. Van Winkelhoff, A.J.; Rams, T.E; Slots, J. (1996). Systemic antibiotic therapy in periodontics. Periodontol 2000. 10, 45-78.

23. Velge, P.; Cloeckaert, A.; Barrow, P. (2005). Emergence of Salmonella epidemics: the problem related to Salmonella enterica serotype enteritidis and multiple antibiotic resistance in other major serotypes. Vet. Res. 36(3), 267-288.

24. Williams, D.W.; Lewis, M.A.O. (2000). Isolation and identification of Candida from the oral cavity. Oral Dis. 6(1), 3-11.

25. World Health Organization. Treatment of tuberculosis: guidelines for national programmes. 3. ed. Geneva: WHO. 2003. Available at: http://www.who.int/gtb/publications. Accessed November 20, 2004.

26. Zöllner, M.S.C.; Jorge, A.O.C. (2003). Candida spp. occurrence in oral cavities of breastfeeding infants and their mother's mouths and breasts. Braz. Dent. Res. 17(2), 151-155. 